\title{
An Efficient Algorithm of Logarithmic Transformation to Hirota Bilinear Form of KdV-type Bilinear Equation
}

\author{
Yichao Ye, Lihong Wang, Zhaowei Chang, Jingsong He* \\ Department of Mathematics, Ningbo University, Ningbo Zhejiang 315211, P.R. China
}

\begin{abstract}
In this paper, an efficient algorithm of logarithmic transformation to Hirota bilinear form of the KdV-type bilinear equation is established. In the algorithm, some properties of Hirota operator and logarithmic transformation are successfully applied, which helps to prove that the linear terms of the nonlinear partial differential equation play a crucial role in finding the Hirota bilinear form. Experimented with various integro-differential equations, our algorithm is proven to be more efficient than the algorithm referred by $\mathrm{Zhou}, \mathrm{Fu}$, and $\mathrm{Li}$ in getting the Hirota bilinear form, especially in achieving the coefficient of the logarithmic transformation.
\end{abstract}

Keywords: Hirota bilinear form, KdV-type bilinear equation, D-operator, Logarithmic transformation, Symbolic computation.

\section{Introduction}

In 1971, Hirota developed a direct method, Hirota bilinear method, to construct the exact multi-soliton solution of integrable nonlinear partial differential equation (NPDE) [1-3]. Once the Hirota bilinear form(HBF) of a NPDE is given, there are several ways to solve it. The solutions of NPDE can be constructed from HBF by the typical perturbation expansion method [1] and others $[4,5]$. Therefore, the key step of the Hirota direct method is to transform the NPDE into the Hirota bilinear form (HBF). Hietarinta designed a program on searching for integrable bilinear equations such as the KdV-type [6], mKdV-type [7], SG-type [8], NLS-type [9] equations. In 1992, Hereman and Zhuang [10] gave a summary on types of bilinear equations. In recent years, some algorithms for generating bilinear form of NPDE are described and Maple packages Bilinearization and HBFTrans are established by Zhou, Fu, Li [11, 12] and Yang, Ruan [13], respectively. The package Bilinearization can construct the HBF of many NPDEs by solving a system of over-determined algebraic equations with respect to the combinatorial coefficients. However, the general ansatz of the bilinear form of NPDE in Ref. [11, 12], which relies on the WTC method [14] and the HBM method [15], is so complicated that the efficiency of performance is relatively low. Without depending on these two methods, our method can obtain the $\mathrm{HBF}$ of the KdV-type equations within shorter time. In this paper, some properties of Hirotaoperator are taken advantage of, which brings forward to a more efficient algorithm for finding

\footnotetext{
*Corresponding author

Email address: hejingsong@nbu.edu.cn (Jingsong He)

Preprint submitted to Applied Mathematics and Computation
}

March 21, 2018 
the HBF of KdV-type equations in NPDE. We take a series of classic KdV-type equations for instance, to demonstrate the validity of our algorithm. Furthermore, the implementation of the algorithm in Maple is applied to automate the tedious computation for the construction of the HBF of KdV-type equations in NPDE.

\section{Hirota Bilinear Method}

Now, let us briefly review the Hirota derivatives. In 1971, Hirota developed the Hirota derivative, which is also called D-operator [1]. For $1+1$ dimensions, the D-operator is defined by

$$
D_{x}^{n} D_{t}^{m} f \cdot g=\left.\left(\partial_{x}-\partial_{x^{\prime}}\right)^{n}\left(\partial_{t}-\partial_{t^{\prime}}\right)^{m} f(x, t) g\left(x^{\prime}, t^{\prime}\right)\right|_{x^{\prime}=x, t^{\prime}=t}, \quad m, n=0,1,2,3, \cdots .
$$

where $f(x, t)$ and $g(x, t)$ are differentiable functions of $x$ and $t$, respectively.

From the definition, there are some properties [1] of the D-operator,

$$
\begin{aligned}
& D_{x}^{n} D_{t}^{m} f \cdot g=D_{t}^{m} D_{x}^{n} f \cdot g=D_{x}^{n-1} D_{t}^{m} D_{x} f \cdot g \\
& D_{x}^{n} D_{t}^{m} f \cdot 1=\partial_{x}^{n} \partial_{t}^{m} f, \quad \text { where } \partial_{x}^{n} \equiv \partial^{n} / \partial x^{n} . \\
& D_{x}^{n} D_{t}^{m} f \cdot g=(-1)^{m+n} D_{x}^{n} D_{t}^{m} g \cdot f \\
& D_{x}^{n} D_{t}^{m} f \cdot f=0, \quad \text { if } m+n \text { is odd; }
\end{aligned}
$$

furthermore, for arbitrary independent variable $x$, a recursive definition of D-operator is,

$$
D_{x}^{n} f \cdot g= \begin{cases}f g, & \text { for } \quad n=0 \\ D_{x}^{n-1} f_{x} \cdot g-D_{x}^{n-1} f \cdot g_{x}, & \text { for } \quad n>0 .\end{cases}
$$

As an example, the Korteweg de Viries (KdV) equation

$$
u_{x x x}+6 u u_{x}+u_{t}=0
$$

where $u=u(x, t)$, can be transformed through the dependent variable transformation,

$$
u=2(\ln f)_{x x}
$$

into

$$
\frac{\partial}{\partial x}\left(\frac{f_{x t} f-f_{x} f_{t}+f_{x x x x} f-4 f_{x x x} f_{x}+3 f_{x x}^{2}}{f^{2}}\right)=0,
$$

from which the bilinear equation below is obtained

$$
f_{x t} f-f_{x} f_{t}+f_{x x x x} f-4 f_{x x x} f_{x}+3 f_{x x}^{2}=c f^{2},
$$

where $\mathrm{c}$ is a constant of integration. Equation 2.10 , with $\mathrm{c}=0$, may also be written concisely in terms of D-operators as

$$
\left(D_{x}^{4}+D_{x} D_{t}\right) f \cdot f=0 .
$$

Thus, the Hirota bilinear form of the KdV equation is obtained. 


\section{Principles Of Our Algorithm}

\subsection{The Properties of Hirota Derivatives}

Introducing vector notation

$$
\vec{D}=\left(D_{t}, D_{x}, D_{y}, \cdots\right),
$$

consider the KdV-type bilinear equation [1]

$$
P\left(D_{t}, D_{x}, D_{y}, \cdots\right) f \cdot f=0,
$$

where $P$ is a general polynomial in $D_{t}, D_{x}, D_{y}, \cdots$.

According to 2.4 and 2.5), it follows that

$$
P(-\vec{D})=P(\vec{D}) .
$$

Now assume that the degree of every term in $P$ is even.

When $n$ is even, it's true that

$$
\begin{aligned}
D_{x}^{n} f \cdot f & =\left.\left(\sum_{i=0}^{n} C_{n}^{i} \partial_{x}^{n-i}\left(-\partial_{x^{\prime}}^{i}\right)\right) f(x) f\left(x^{\prime}\right)\right|_{x^{\prime}=x} \\
& =\sum_{i=0}^{n}(-1)^{i} C_{n}^{i}\left(\partial_{x}^{n-i} f(x)\right)\left(\partial_{x}^{i} f(x)\right) \\
& =2 \sum_{i=0}^{n / 2-1}(-1)^{i} C_{n}^{i}\left(\partial_{x}^{n-i} f(x)\right)\left(\partial_{x}^{i} f(x)\right)+(-1)^{n / 2} C_{n}^{n / 2}\left(\partial_{x}^{n / 2} f(x)\right)^{2} \\
& =2\left(\partial_{x}^{n} f\right) f+H,
\end{aligned}
$$

where $H=H\left(f_{x}, f_{t}, \cdots\right)$ is a polynomial in $f_{x}, f_{t}, \cdots$, WITHOUT $f$, and $H=D_{x}^{n} f \cdot f-2\left(\partial_{x}^{n} f\right) f$. This simple observation implies the following crucial formula

$$
P\left(D_{t}, D_{x}, D_{y}, \cdots\right) f \cdot f=2\left(P\left(\partial_{t}, \partial_{x}, \partial_{y}, \cdots\right) f\right) f+\widetilde{H}=2(P(\vec{\partial}) f) f+\widetilde{H},
$$

where $\widetilde{H}$ is a polynomial in $f_{x}, f_{t}, \cdots$, WITHOUT $f$, and $P(\vec{\partial})=P\left(\partial_{t}, \partial_{x}, \partial_{y}, \cdots\right)$. Some simple examples are listed as follows to illustrate equation (3.5)

$$
\begin{aligned}
& D_{x}^{2} f \cdot f=2\left(f_{x x} f-f_{x}^{2}\right), \quad \widetilde{H}=-2 f_{x}^{2}, \\
& D_{x}^{4} f \cdot f=2\left(f_{4 x} f-4 f_{3 x} f_{x}+3 f_{x x}^{2}\right), \quad \widetilde{H}=-8 f_{3 x} f_{x}+6 f_{x x}^{2}, \\
& D_{x} D_{t} f \cdot f=2\left(f_{t x} f-f_{t} f_{x}\right), \quad \widetilde{H}=-2 f_{t} f_{x},
\end{aligned}
$$

where $f_{k x} \equiv \partial_{x}^{k} f \equiv \frac{\partial^{k} f}{\partial x^{k}}, k \in \mathbb{N}$. Furthermore, using equation (3.5), it is easy to find $P(\vec{D}) f \cdot f$ is a quadratic polynomial in $f, f_{x}, f_{t}, \cdots$, and then to obtain

$$
\frac{P(\vec{D}) f \cdot f}{f^{2}}=\frac{P_{1}}{f}+\frac{P_{2}}{f^{2}}
$$

Here $P_{1}$ and $P_{2}$ are polynomials in $f_{x}, f_{t}, \cdots$, WITHOUT $f . P_{1}=2 P(\vec{\partial}) f$, is a linear polynomial; $P_{2}=\widetilde{H}$ is a quadratic polynomial. 


\subsection{Logarithmic Transformation}

Consider the derivation formula,

$$
\left(\frac{f}{g}\right)_{x}=\frac{f_{x} g-f g_{x}}{g^{2}}=\frac{f_{x}}{g}-\frac{f g_{x}}{g^{2}}
$$

Furthermore, for arbitrary independent variable $t$, it's easy to show that

$$
\left(\frac{f_{x}}{f}\right)_{k t}=\frac{\bar{P}_{1}}{f}+\frac{\bar{P}_{2}}{f^{2}}+\cdots+\frac{\bar{P}_{k+1}}{f^{k+1}}
$$

where $k \in \mathbb{N}$ and $\bar{P}_{i}(i=1,2, \cdots, k+1)$ is a homogeneous polynomial of degree $i$ in $f_{x}, f_{t}, \cdots$, WITHOUT $f$, and

$$
\bar{P}_{1}=\partial_{t}^{k} f_{x}, \bar{P}_{k+1}=(-1)^{k} k ! f_{x} f_{t}^{k} .
$$

are obtained by using equation (3.10) repeatedly.

Furthermore, when $m+n \geq 1$, it shows that

$$
\partial_{x}^{n} \partial_{t}^{m}(\ln f)=\frac{\partial_{x}^{n} \partial_{t}^{m} f}{f}+\cdots+(-1)^{m+n-1}(m+n-1) ! \frac{f_{x}^{n} f_{t}^{m}}{f^{m+n}}
$$

Still, by using $P$ as the denotation of a general polynomial and letting the lowest degree of the terms in $P$ be equal to or greater than 1, we have

$$
P(\vec{\partial})(\ln f)=\frac{\widetilde{P}_{1}}{f}+\frac{\widetilde{P}_{2}}{f^{2}}+\cdots+\frac{\widetilde{P}_{r}}{f^{r}}
$$

where $r=\operatorname{degree}(P)$ and $\widetilde{P}_{1}=P(\vec{\partial}) f$. Here, $\widetilde{P}_{i}(i=1,2, \cdots, r)$ also denotes a homogeneous polynomial of degree $i$ in $f_{x}, f_{t}, \cdots$, WITHOUT $f$.

Using the logarithmic transformation $u=2 \ln f$, the following expressions are obtained:

$$
\begin{aligned}
& u_{x x}=2\left(\frac{f_{x x}}{f}-\frac{f_{x}^{2}}{f^{2}}\right)=\frac{D_{x}^{2} f \cdot f}{f^{2}} \\
& u_{x t}=2\left(\frac{f_{x t}}{f}-\frac{f_{x} f_{t}}{f^{2}}\right)=\frac{D_{x} D_{t} f \cdot f}{f^{2}} \\
& u_{t t}=2\left(\frac{f_{t t}}{f}-\frac{f_{t}^{2}}{f^{2}}\right)=\frac{D_{t}^{2} f \cdot f}{f^{2}} \\
& u_{4 x}=2\left(\frac{f_{x x x x}}{f}-\frac{4 f_{x x x} f_{x}+3 f_{x, x}^{2}}{f^{2}}+\frac{12 f_{x x} f_{x}^{2}}{f^{3}}-\frac{6 f_{x}^{4}}{f^{4}}\right)=\frac{D_{x}^{4} f \cdot f}{f^{2}}-3\left(\frac{D_{x}^{2} f \cdot f}{f^{2}}\right)^{2} \\
& u_{6 x}=2\left(\frac{f_{x x x x x x}}{f}+\cdots-\frac{120 f_{x}^{6}}{f^{6}}\right)=\frac{D_{x}^{6} f \cdot f}{f^{2}}-15 \frac{D_{x}^{4} f \cdot f}{f^{2}} \frac{D_{x}^{2} f \cdot f}{f^{2}}+30\left(\frac{D_{x}^{2} f \cdot f}{f^{2}}\right)^{3} .
\end{aligned}
$$

Clearly, the expressions above can lead us to finding the KdV-type bilinear form associated with logarithmic transformation in NPDE. 


\subsection{The Relationship Between Logarithmic Transformation and KdV-type Bilinear Equation}

In this paper, we consider the logarithmic transformation

$$
u=2 \alpha(\ln f)_{n x}, \quad n=0,1,2
$$

where $f=f(x, t, \cdots)$ and $\alpha$ is a nonzero constant.

Substituting (3.20) to a NPDE

$$
W\left(u, u_{x}, u_{t}, \cdots\right)=0
$$

we get

$$
\left.\widetilde{W}\left(f, f_{x}, f_{t}, \cdots ; \alpha\right) \equiv W\left(u, u_{x}, u_{t}, \cdots\right)\right|_{u=2 \alpha(\ln f)_{n x}} .
$$

If NPDE $\widetilde{W}\left(f, f_{x}, f_{t}, \cdots ; \alpha\right)$ has the $\mathrm{KdV}$-type bilinear form

$$
\left(\frac{P(\vec{D}) f \cdot f}{f^{2}}\right)_{m x}=0,
$$

where $m$ is a nonnegative integer, using the properties of Hirota operator and logarithmic transformation, our algorithm will find the undetermined parameters $n, m, P(\vec{D})$, and $\alpha$.

Because of 3.14, the terms in NPDE with degree $k$ generate the homogeneous expression with logarithmic transformation 3.20)

$$
\frac{\widetilde{P}_{k}}{f^{k}}+\frac{\widetilde{P}_{k+1}}{f^{k+1}}+\cdots .
$$

That is, with logarithmic transformation 3.20), the terms in NPDE with degree $k$ do NOT generate the terms $\frac{\widetilde{P}_{i}}{f^{i}}(i=1,2,3, \cdots, k-1)$.

Consider KdV-type bilinear form

$$
\begin{aligned}
& \left(\frac{P(\vec{D}) f \cdot f}{f^{2}}\right)_{m x}=\left(\frac{P_{1} f+P_{2}}{f^{2}}\right)_{m x}=\left(\frac{P_{1}}{f}+\frac{P_{2}}{f^{2}}\right)_{m x}=\left(\frac{P_{1}}{f}\right)_{m x}+\left(\frac{P_{2}}{f^{2}}\right)_{m x} \\
= & \left(\frac{P_{1 m x}}{f}+\cdots+\frac{(-1)^{m} m ! P_{1} f_{x}^{m}}{f^{m+1}}\right)+\left(\frac{P_{2 m x}}{f^{2}}+\cdots+\frac{(-1)^{m}(m+1) ! P_{2} f_{x}^{m}}{f^{m+2}}\right) \\
= & \frac{P_{1 m x}}{f}+\cdots+\frac{(-1)^{m}(m+1) ! P_{2} f_{x}^{m}}{f^{m+2}}=\frac{2 \partial_{x}^{m}(P(\vec{\partial}) f)}{f}+\cdots+\frac{(-1)^{m}(m+1) ! P_{2} f_{x}^{m}}{f^{m+2}} .
\end{aligned}
$$

Therefore, if a equation has the KdV-type bilinear form, with logarithmic transformation (3.20), the term $\frac{P_{1 m x}}{f}$ must be generated from the linear terms in NPDE 3.21.

Denoting the linear part in NPDE (3.21) by $P_{l}(\vec{\partial}) u$, with logarithmic transformation (3.20), according to 3.14), the linear part is transformed into

$$
P_{l}(\vec{\partial}) u \stackrel{u=2 \alpha(\ln f)_{n x}}{\longrightarrow} \frac{2 \alpha \partial_{x}^{n} P_{l}(\vec{\partial}) f}{f}+\cdots
$$

Thus, it follows that

$$
P(\vec{\partial})=\alpha \partial_{x}^{n-m} P_{l}(\vec{\partial})
$$


From the above equation, we find it important that the linear terms of the NPDE play a crucial role in finding the HBF. Hence, from the linear part in NPDE, $P$, the function of HBF, can be obtained.

Let

$$
\left(\frac{P(\mathbf{D}) f \cdot f}{f^{2}}\right)_{m x}=\widetilde{W}\left(f, f_{x}, f_{t}, \cdots ; \alpha\right),
$$

then the difference should be zero, namely,

$$
\operatorname{Res}_{\widetilde{W}} \triangleq \widetilde{W}\left(f, f_{x}, f_{t}, \cdots ; \alpha\right)-\left(\frac{P(\vec{D}) f \cdot f}{f^{2}}\right)_{m x}=0 .
$$

Clearly, $n$ is a finite enumerated integer and $m$ is also a finite enumerated integer (See Step 5 of algorithm in Section 5) and $P(\vec{\partial})=\alpha \partial_{x}^{n-m} P_{l}(\vec{\partial})$.

With $P$ satisfying (3.26), the coefficient of $\frac{1}{f}$ in $\operatorname{Res}_{\widetilde{W}}$ is equal to zero. Then the homogeneous expression in $f$ and its derivatives follows as:

$$
\operatorname{Res}_{\widetilde{W}}=\frac{\widehat{P}_{2}}{f^{2}}+\cdots+\frac{\widehat{P}_{r}}{f^{r}} .
$$

Here, $r$ is a finite positive integer.

Solving the numerators of 3.29), if there exists the nonzero numerical solution $\alpha$, the undetermined parameters $n, m, P(\vec{D})$ are also achieved. It can be proven that $\alpha$ has no more than one nonzero numerical solution which satisfies 3.28 with certain $n$ and $m$. The proof will be given in our next paper.

In summary, if a NPDE has the KdV-type bilinear form associated with the logarithmic transformation 3.20, our algorithm will surely find it.

\section{An Example Of our Algorithm}

Now, let us take the KdV equation as an example.

$$
u_{x x x}+6 u u_{x}+u_{t}=0 .
$$

Here, $P_{l}(\vec{\partial})=\partial_{t}+\partial_{x}^{3}$.

First substitute $u=2 \alpha \ln f$ to 4.1) and simplify the equation, the homogeneous equation in $f$ and its derivatives follows as:

$$
\frac{2 \alpha\left(f_{x x x}+12 \alpha(\ln f) f_{x}+f_{t}\right)}{f}-\frac{6 \alpha f_{x x} f_{x}}{f^{2}}+\frac{4 \alpha f_{x}^{3}}{f^{3}}=0 .
$$

Since the above equation has $\ln (f)$ term, we goto the next logarithmic transformation $u=$ $2 \alpha(\ln f)_{x}$.

Likewise, substitute $u=2 \alpha(\ln f)_{x}$ to (4.1) and simplify the equation, the homogeneous equation in $f$ and its derivatives follows as:

$$
\frac{2 \alpha\left(f_{x x x x}+f_{t x}\right)}{f}-\frac{2 \alpha\left(-12 \alpha f_{x x} f_{x}+4 f_{x x x} f_{x}+3 f_{x x}^{2}+f_{x} f_{t}\right)}{f^{2}}+\frac{24 \alpha f_{x}^{2}\left(-\alpha f_{x}+f_{x x}\right)}{f^{3}}-\frac{12 \alpha f_{x}^{4}}{f^{4}}=0 .
$$


From the above equation, the coefficient of $\frac{1}{f}$ is acquired: $P_{1}=2 \alpha\left(f_{x x x x}+f_{t x}\right)=$ $2 \alpha\left(\partial_{x}^{4}+\partial_{x} \partial_{t}\right) f=2 \alpha \partial_{x} P_{l}(\vec{\partial}) f$. Because the order of derivative is even, the Hirota bilinear form can be obtained: $P(\vec{D}) f \cdot f=\alpha\left(D_{x}^{4}+D_{x} D_{t}\right) f \cdot f$, with which divided by $f^{2}$ and subtracts the above equation the difference is as follows:

$$
\frac{12 f_{x x} \alpha\left(-2 \alpha f_{x}+f_{x x}\right)}{f^{2}}-\frac{24 \alpha f_{x}^{2}\left(-\alpha f_{x}+f_{x x}\right)}{f^{3}}+\frac{12 \alpha f_{x}^{4}}{f^{4}}=0 .
$$

Solving the numerators of the difference equation, it only has $\alpha=0$. So we goto the next logarithmic transformation $u=2 \alpha(\ln f)_{x x}$.

Similarly, substitute $u=2 \alpha(\ln f)_{x x}$ to 4.1 and simplify the equation, the homogeneous equation in $f$ and its derivatives follows as:

$$
\begin{gathered}
\frac{2 \alpha\left(f_{t x x}+f_{x x x x x}\right)}{f}-\frac{2 \alpha\left(-12 \alpha f_{x x x} f_{x x}+f_{x x} f_{t}+5 f_{x x x x} f_{x}+10 f_{x x x} f_{x x}\right)+2 f_{x} f_{t x}}{f^{2}} \\
-\frac{4 f_{x} \alpha\left(18 \alpha f_{x x}^{2}+6 \alpha f_{x x x} f_{x}-15 f_{x x}^{2}-10 f_{x x x} f_{x}-f_{x} f_{t}\right)}{f^{3}}+\frac{120 \alpha f_{x x} f_{x}^{3}(\alpha-1)}{f^{4}}-\frac{48 \alpha f_{x}^{5}(\alpha-1)}{f^{5}}=0 .
\end{gathered}
$$

From the above equation, the coefficient of $\frac{1}{f}$ is obtained: $P_{1}=2 \alpha\left(f_{x x x x x}+f_{t x x}\right)=$ $2 \alpha\left(\partial_{x}^{5}+\partial_{x}^{2} \partial_{t}\right) f$. Because the order of derivative is odd, we integrate $P_{1}$ and get the Hirota bilinear form $P(\vec{D}) f \cdot f=\alpha\left(D_{x}^{4}+D_{x} D_{t}\right) f \cdot f$, with which divided by $f^{2}$ and derived in terms of $x$ one time then subtracts the above equation, the difference is obtained:

$$
\frac{-24 \alpha f_{x x x} f_{x x}(\alpha-1)}{f^{2}}+\frac{24 f_{x} \alpha(\alpha-1)\left(3 f_{x x}^{2}+f_{x x x} f_{x}\right)}{f^{3}}-\frac{120 \alpha f_{x x} f_{x}^{3}(\alpha-1)}{f^{4}}+\frac{48 \alpha f_{x}^{5}(\alpha-1)}{f^{5}}=0 .
$$

Solving the numerators of the difference equation, a nonzero solution, $\alpha=1$, is achieved.

In conclusion, by using the logarithmic transformation $u=2(\ln f)_{x x}$, it shows that the equation (4.1) is equivalent to

$$
\left(\frac{\left(D_{x}^{4}+D_{x} D_{t}\right) f \cdot f}{f^{2}}\right)_{x}=0 .
$$

This example illustrates our method intuitively.

\section{An Algorithm of Bilinear}

Consider the general NPDE

$$
W\left(u, u_{x}, u_{t}, \cdots\right)=0,
$$

where $u=u(x, t, \cdots)$, and $W$ is a polynomial in $u$ and its derivatives.

The algorithm for obtaining HBF goes in the following steps:

Step 1 Let $n=0, f=f(x, t, \cdots)$ and $\alpha$ is an undetermined nonzero constant.

Step 2 Using logarithmic transformation $u=2 \alpha(\ln f)_{n x}$, it follows that

$$
\widetilde{W}=\widetilde{W}\left(f, f_{x}, f_{t}, \cdots\right)=\left.W\left(u, u_{x}, u_{t}, \cdots\right)\right|_{u=2 \alpha(\ln f)_{n x}}
$$


Step 3 If $\widetilde{W}$ has $\ln (f)$ term, then goto Step 6.

Step 4 Simplify the equation $\widetilde{W}$ to get the homogeneous expressions in $f$ and its derivatives

$$
\widetilde{W}=\frac{P_{1}}{f}+\frac{P_{2}}{f^{2}}+\cdots+\frac{P_{r}}{f^{r}},
$$

where $P_{i}=P_{i}\left(f_{x}, f_{t}, \cdots\right)(i=1,2, \cdots, r)$ is a homogeneous polynomial of degree $i$ in $f_{x}, f_{t}, \cdots$, WITHOUT $f$.

Step 5 Get the coefficient of $\frac{1}{f}$ in $\widetilde{W}: P_{1}=P_{1}\left(f_{x}, f_{t}, \cdots\right)$. Let the lowest order of the derivative of $f$ with respect to $x$ in $P_{1}$ is $k$, and the corresponding Hirota bilinear form is $P\left(D_{x}, D_{t}, \cdots\right) f \cdot f$. Then the following equation is obtained:

$$
P_{1}\left(f_{x}, f_{t}, \cdots\right)=2 \partial_{x}^{m} P\left(f_{x}, f_{t}, \cdots\right),
$$

where $0 \leq m \leq k$ and the degrees of the terms in $P\left(f_{x}, f_{t}, \cdots\right)$ are even. That is,

- If the lowest order derivative of $f$ is even, then $m=0,2,4, \cdots$, and $m \leq k$.

- If the lowest order derivative of $f$ is odd, then $m=1,3,5, \cdots$, and $m \leq k$.

Step 5.1 Let $m=0$, if the lowest order of the derivative of $f$ is even, or else let $m=1$.

Step 5.2 Integrating $P_{1}$ by $m$ times in terms of $x$, we get $P\left(f_{x}, f_{t}, \cdots\right)=\frac{1}{2} \partial_{x}^{-m} P_{1}\left(f_{x}, f_{t}, \cdots\right)$. Hence the $\operatorname{HBF}$ is $P\left(D_{x}, D_{t}, \cdots\right) f \cdot f$.

Step 5.3 Calculate the difference, $\operatorname{Res}_{\widetilde{W}}=\widetilde{W}-\left(\frac{P\left(D_{x}, D_{t}, \cdots\right) f \cdot f}{f^{2}}\right)_{m x}$, and simplify the difference $\operatorname{Res}_{\widetilde{W}}$ to get the homogeneous expressions in $f$ and its derivatives

$$
\operatorname{Res}_{\widetilde{W}}=\frac{\widehat{P}_{2}}{f^{2}}+\cdots+\frac{\widehat{P}_{r}}{f^{r}} .
$$

Step 5.4 Get the equations $\widehat{P}_{i}=0(i=2,3, \cdots, r)$, and solve the equations. If a nonzero $\alpha$ exists, then using $u=2 \alpha(\ln f)_{n x}$, the NPDE is equal to $\left(\frac{P\left(D_{x}, D_{t}, \cdots\right) f \cdot f}{f^{2}}\right)_{m x}=0$. Therefore, the $\mathrm{HBF}$ is $P\left(D_{x}, D_{t}, \cdots\right) f \cdot f$, and exit of program.

Step 5.5 Let $m=m+2$. If $m \leq k$, goto Step 5.2, or else goto Step 6.

Step 6 Let $n=n+1$. If $n \leq 2$ then goto Step 2, or else the NPDE has no HBF with logarithmic transformation, end program and exit.

\section{Applications}

Example 1. Boussinesq equation [16, 17]

$$
u_{t t}-u_{x x}-3 u_{x x}^{2}-u_{4 x}=0
$$

Solution. From the equation, Bilinear within 0.s outputs $\alpha=1, u=2 \ln (f)$ and

$$
\frac{\left(D_{t}^{2}-D_{x}^{2}-D_{x}^{4}\right) f \cdot f}{f^{2}}=0 .
$$


Example 2. Sawada-Kotera equation $\lfloor 18,19]$

$$
u_{t}+45 u_{x} u^{2}-15 u_{x x} u_{x}-15 u_{x x x} u+u_{5 x}=0
$$

Solution. Within 0.032 s our program outputs $\alpha=-1, u=-2(\ln f)_{x x}$ and

$$
\left(\frac{\left(-D_{x}^{6}-D_{x} D_{t}\right) f \cdot f}{f^{2}}\right)_{x}=0
$$

Example 3. Kadomtsev-Petviashvili equation [20]

$$
\left(u_{3 x}+6 u u_{x}+u_{t}\right)_{x}+3 \delta^{2} u_{y y}=0
$$

Solution. Bilinear within 0.016 s outputs $\alpha=1, u=2(\ln f)_{x x}$ and

$$
\left(\frac{\left(D_{x}^{4}+D_{x} D_{t}+3 \delta^{2} D_{y}^{2}\right) f \cdot f}{f^{2}}\right)_{x x}=0 .
$$

Example 4. The shallow water waves equation [21]

$$
u_{t}-u_{x x t}-3 u u_{t}+3 u_{x} \int_{x}^{\infty} u_{t} d x+u_{x}=0
$$

Solution. By substituting $u=w_{x}$ and using the boundary condition $\left.u_{t}\right|_{x \rightarrow \infty}=0$ 6.4 can be converted into the differential form

$$
w_{x t}-w_{3 x t}-3 w_{x} w_{x t}-3 w_{x x} w_{t}+w_{x x}=0,
$$

Bilinear within 0.016 s outputs $\alpha=1, w=2(\ln f)_{x}$ and

$$
\left(\frac{\left(-D_{x}^{3} D_{t}+D_{x}^{2}+D_{x} D_{t}\right) f \cdot f}{f^{2}}\right)_{x}=0 .
$$

Example 5. Ito equation [22]

$$
u_{t t}+u_{3 x t}+6 u_{x} u_{t}+3 u u_{x t}+3 u_{x x} \int_{-\infty}^{x} u_{t} d x=0
$$

Solution. By substituting $u=w_{x}$ and using the boundary condition $\left.u_{t}\right|_{x \rightarrow-\infty}=0$ (6.6) is transformed into the differential form

$$
w_{x t t}+w_{4 x t}+6 w_{x x} w_{x t}+3 w_{x} w_{x x t}+3 w_{3 x} w_{t}=0,
$$

Bilinear within 0.031 s outputs $\alpha=1, w=2(\ln f)_{x}$ and

$$
\left(\frac{\left(D_{x}^{3} D_{t}+D_{t}^{2}\right) f \cdot f}{f^{2}}\right)_{x x}=0 .
$$

Example 6. $(2+1)$-dimensional breaking soliton equation [23]

$$
u_{t}+\beta u_{x x y}+4 \beta u u_{y}+4 \beta u_{x} \int_{-\infty}^{x} u_{y} d x=0
$$


Solution. The equation can be written as (6.9) by substituting $u=w_{x}$ and using the boundary condition $\left.u_{y}\right|_{x \rightarrow-\infty}=0$

$$
w_{x t}+\beta w_{3 x y}+4 \beta w_{x} w_{x y}+4 \beta w_{x x} w_{y}=0
$$

Bilinear within 0.016 s outputs $\alpha=\frac{3}{4}, w=\frac{3}{2}(\ln f)_{x}$ and

$$
\left(\frac{3}{4} \frac{\left(D_{x} D_{t}+\beta D_{x}^{3} D_{y}\right) f \cdot f}{f^{2}}\right)_{x}=0 .
$$

Example 7. The Bidirectional SK equation [24]

$$
5 \int_{-\infty}^{x} u_{t t} d x+5 u_{x x t}-15 u u_{t}-15 u_{x} \int_{-\infty}^{x} u_{t} d x-45 u_{x} u^{2}+15 u_{x x} u_{x}+15 u_{3 x} u-u_{5 x}=0
$$

Solution. Similarly, we convert 6.10 into the differential form by substituting $u=w_{x}$ and using the boundary condition $\left.u_{t}\right|_{x \rightarrow-\infty}=0,\left.u_{t t}\right|_{x \rightarrow-\infty}=0$

$$
5 w_{t t}+5 w_{3 x t}-15 w_{x} w_{x t}-15 w_{x x} w_{t}-45 w_{x x} w_{x}^{2}+15 w_{3 x} w_{x x}+15 w_{4 x} w_{x}-w_{6 x}=0,
$$

Bilinear within 0.015 s outputs $\alpha=-1, w=-2(\ln f)_{x}$ and

$$
\left(\frac{\left(-5 D_{t}^{2}+D_{x}^{6}-5 D_{x}^{3} D_{t}\right) f \cdot f}{f^{2}}\right)_{x}=0 .
$$

Example 8. $(2+1)$-dimensional SK equation [25]

$$
9 u_{t}+u_{5 x}+15 u_{x x} u_{x}+15 u_{3 x} u+45 u_{x} u^{2}-5 \int_{-\infty}^{x} u_{y y} d x-15 u u_{y}-15 u_{x} \int_{-\infty}^{x} u_{y} d x-5 u_{x x y}=0
$$

Solution. With the substitution $u=w_{x}$ and the boundary condition $\left.u_{y}\right|_{x \rightarrow-\infty}=0,\left.u_{y y}\right|_{x \rightarrow-\infty}=0$ (6.12) acquires a differential form

$$
9 w_{x t}+w_{6 x}+15 w_{3 x} w_{x x}+15 w_{4 x} w_{x}+45 w_{x x} w_{x}^{2}-5 w_{y y}-15 w_{x} w_{x y}-15 w_{x x} w_{y}-5 w_{3 x y}=0,
$$

Bilinear within 0.032 s outputs $\alpha=1, w=2(\ln f)_{x}$ and

$$
\left(\frac{\left(D_{x}^{6}-5 D_{x}^{3} D_{y}-5 D_{y}^{2}+9 D_{x} D_{t}\right) f \cdot f}{f^{2}}\right)_{x}=0 .
$$

Example 9. (3+1)-dimensional $\mathrm{KdV}$ equation [13]

$$
u_{t}+6 u_{x} u_{y}+u_{x x y}+u_{4 x z}+60 u_{x}^{2} u_{z}+10 u_{3 x} u_{z}+20 u_{x} u_{x x z}=0
$$

Solution. Bilinear within 0.016 s outputs $\alpha=\frac{1}{2}, u=(\ln f)_{x}$ and

$$
\frac{1}{2} \frac{\left(D_{x} D_{t}+D_{x}^{5} D_{z}+D_{x}^{3} D_{y}\right) f \cdot f}{f^{2}}=0 .
$$




\section{The Program Code of Bilinear}

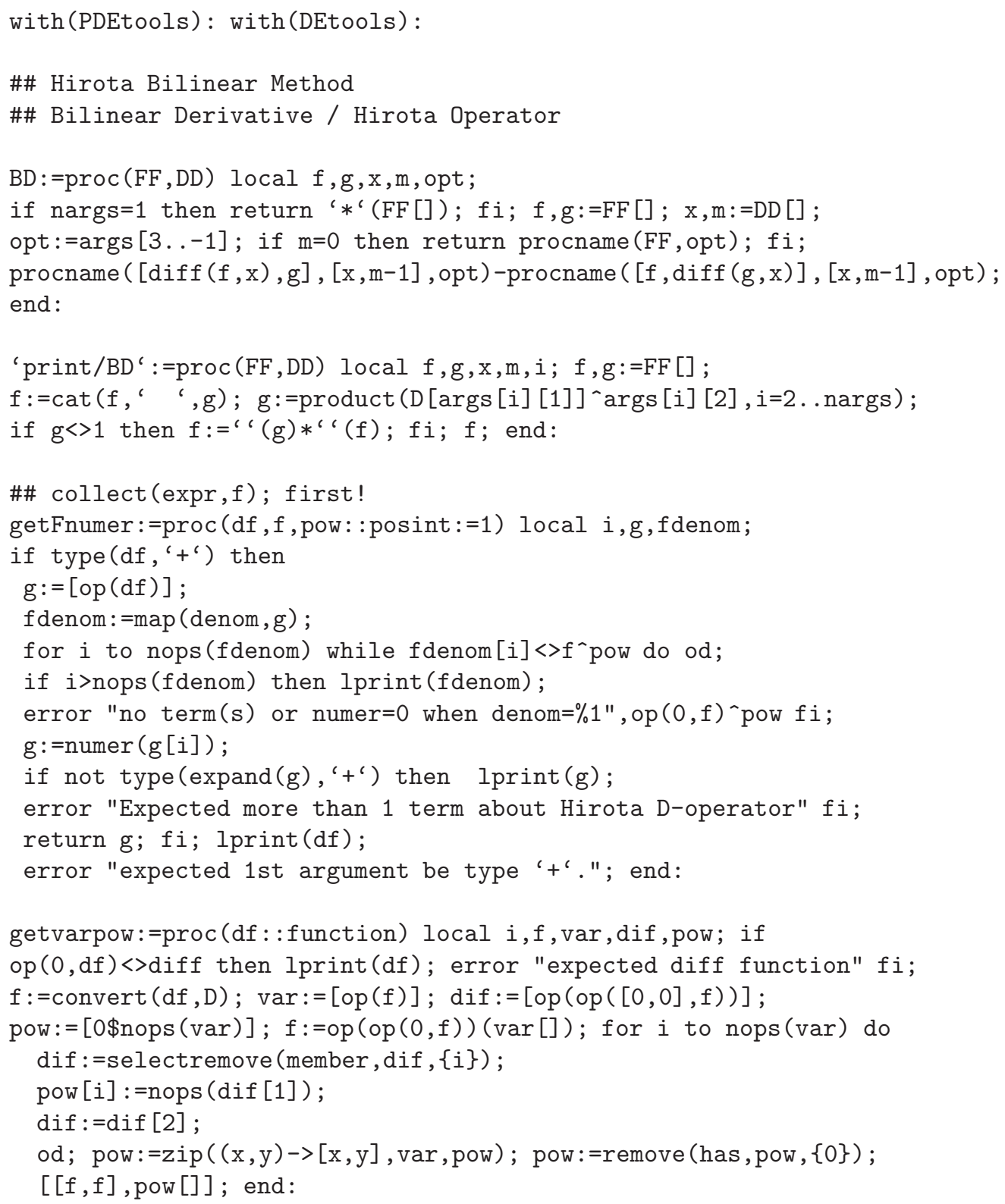




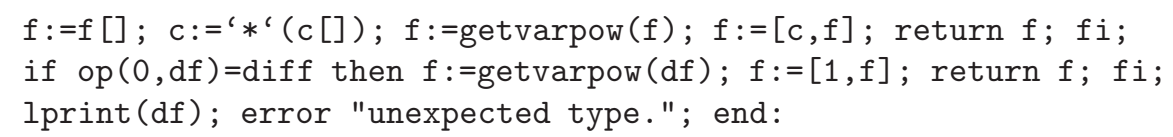

Bilinear:=proc(uf,u,f, x, alpha) local su, h,i,j,g1,CB, PL, gdo, DD, Res ; if hasfun(uf,int) then error "Do not support integral function yet. 


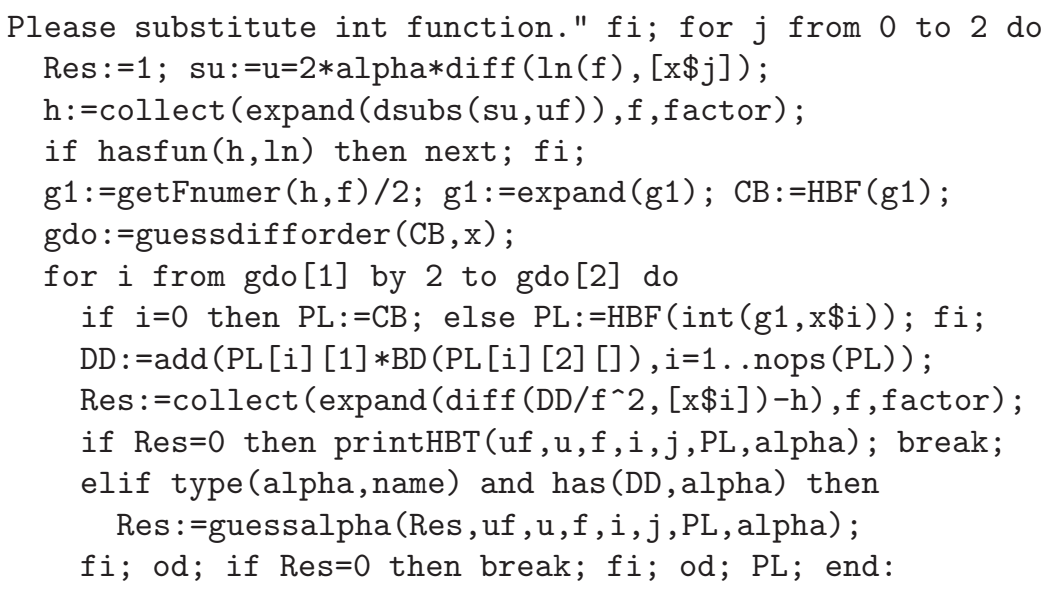

\section{Conclusions}

To sum up, an algorithm for generating the Hirota bilinear form of NPDE with logarithm transformation has been proposed in this paper, and the bilinear forms of a class of NPDEs are obtained by using package Bilinear. Then we illuminate the availability of the algorithm by illustrating some examples.

\section{Acknowledgement}

The work has been partially supported by the Natural Science Foundation of China ( No. 10971109), K.C.Wong Magna Fund in Ningbo University and Student Research of Ningbo University. Jingsong He is also supported by Program for NCET under Grant No.NCET-08-0515.

\section{References}

[1] R. Hirota, The direct method in soliton theory (Cambridge University Press, Cambridge, 2004).

[2] A. Scott, Encyclopedia of Nonlinear Science, Taylor and Francis, Routledge, New York, 2005.

[3] Wenxiu Ma, Ruguang Zhou, Liang Gao, Exact one-periodic and two-periodic wave solutions to Hirota bilinear equations in (2+1) dimensions, Mod. Phys. Lett. A 21 (2009) 1677-1688.

[4] Wenxiu Ma, Engui Fan, Linear superposition principle applying to Hirota bilinear equations, Comput. Math. Appl. 61 (2011) 950-959.

[5] J. Hietarinta, Introduction to the Hirota bilinear method. Integrability of nonliear systems (Pondicherry,1996) 95103, Lecture Notes in Physics, 495 (Springer, Berlin, 1997).

[6] J. Hietarinta, A search for bilinear equations passing Hirota's three-soliton condition. I. KdV-type bilinear equations. J. Math. Phys. 28 (1987) 1732-1742.

[7] J. Hietarinta, A search for bilinear equations passing Hirota's three-soliton condition. II. mKdV-type bilinear equations. J. Math. Phys. 28 (1987) 2094-2101.

[8] J. Hietarinta, A search for bilinear equations passing Hirota's three-soliton condition. III. Sine-Gordon-type bilinear equations. J. Math. Phys. 28 (1987) 2586-2592.

[9] J. Hietarinta, A search for bilinear equations passing Hirota's three-soliton condition. IV. Complex bilinear equations. J. Math. Phys. 29 (1988) 628-635.

[10] W. Hereman and W. Zhuang, Symbolic computation of solitons with Macsyma. Computational and applied mathematics, II (Dublin, 1991), 287-296 (North-Holland, Amsterdam, 1992).

[11] Zhenjiang Zhou, Jingzhi Fu and Zhibin Li, An implementation for the algorithm of Hirota bilinear form of PDE in the Maple system. Appl. Math. Comput. 183 (2006) 872-877. 
[12] Zhenjiang Zhou, Jingzhi Fu and Zhibin Li, Maple packages for computing Hirota's bilinear equation and multisoliton solutions of nonlinear evolution equations. Appl. Math. Comput. (2010) 92-104.

[13] Xudong Yang and Hangyu Ruan, A Maple package on symbolic computation of Hirota bilinear form for nonlinear equations. Commun. Theor. Phys. (Beijing, China) 52 (2009) 801-807.

[14] J. Weiss, M. Tabor and G. Carnevale, The Painleve property for partial differential equations. J. Math. Phys. 24 (1983) 522-526.

[15] Mingliang Wang, Yubin Zhou and Zhibin Li, Application of a homogeneous balance method to exact solutions of nonlinear equations in mathematical physics. Phys. Lett. A 216 (1996) 67-75.

[16] G. B. Whitham, Linear and Nonlinear Waves (New York, Wiley, 1974) 9.

[17] D. Zwillinger, Handbook of Differential Equations, 3rd ed. (Boston, MA: Academic Press,1997) 129-130.

[18] K. Sawada and T. Kotera, A method for finding $N$-soliton solutions of the K.d.V. equation and K.d.V.-like equation. Progr. Theoret. Phys. 51 (1974) 1355-1367.

[19] P. J. Caudrey, R. K. Dodd and J. D. Gibbon, A new hierarchy of Korteweg-de Vries equations. Proc. Roy. Soc. London Ser. A 351 (1976) 407-422.

[20] B. B. Kadomtsev and V. I. Petviashvili, On the stability of solitary waves in weakly dispersive media. Sov. Phys. Dokl. 15 (1970) 539-541.

[21] R. Hirota and J. Satsuma, $N$-soliton solutions of model equations for shallow water waves. J. Phys. Soc. Japan. 40 (1976) 611-612.

[22] M. Ito, An extension of nonlinear evolution equations of the K-dV (mK-dV) type to higher orders. J. Phys. Soc. Japan. 49 (1980) 771-778.

[23] WenHua Huang, YuLu Liu and JieFang Zhang, Doubly periodic propagating wave for (2+1)-dimensional breaking soliton equation. Commun. Theor. Phys. (Beijing) 49 (2008) 268-274.

[24] J. M. Dye and A. Parker, A bidirectional Kaup-Kupershmidt equation and directionally dependent solitons. J. Math. Phys. 43 (2002) 4921-4949.

[25] Jingsong He and Xiaodong Li, Solutions of the $(2+1)$-dimensional KP, SK and KK equations generated by gauge transformations from nonzero seeds. J. Nonlinear Math. Phys. 16 (2009) 179-194. 